\title{
Introducing the Urban Community Health Center (UCHC) as a nascent local model: Will it be a linchpin in the health sector reform in Iran?
}

\author{
Mohammad Hadi Imanieh ${ }^{1}$, Ahmad Kalateh Sadati ${ }^{*}$, Mohse \\ *Correspondence to: Ahmad Kalateh Sadati, Email: asadati@sums.ac.ir \\ Copyright: (๑) 2015 by Kerman University of Medical Sciences \\ Citation: Imanieh MH, Sadati AK, Moghadami M, Hemmati A. Introducing \\ the Urban Community Health Center (UCHC) as a nascent local model: Will it be a \\ linchpin in the health sector reform in Iran? Int J Health Policy Manag 2015; 4: 331-332. \\ doi: 10.15171/ijhpm.2015.74 \\ Received: 21 January 2015, Accepted: 30 March 2015, ePublished: 2 April 2015
}

\section{Dear Editor,}

Over the past three decades, the Iranian health system has undergone a wide range of reforms. This period has been associated with changes that have helped improve Iran's health system. The most visible breakthrough has been the establishment and improvement of Primary Health Care (PHC) with an emphasis on rural regions (i.e. rural health centers and rural Health Houses), which represented a condition highlighting the potentials of Behvarz (i.e. community health worker) in PHC. The PHC model is composed of eight elements including: health education, vaccination, mother and child care, nutrition, environmental health, control of endemic and common diseases, epidemic control, and essential drugs. This period has led to remarkable achievements in various areas from prevention to treatment $(1,2)$.

Similar to health systems in other countries, the Iranian health system is also facing some challenges. The growing population, increased urbanization, and broad social changes are accompanied with considerable transformations which, in turn, have caused several problems in the health system. Some of the most important challenges include the increase in the rate of Non-Communicable Diseases (NCDs) (3), aging (4), mental health (5), and AIDS (6). These changes have led many policy-makers to focus attention on structural reforms in the health system because goal-driven reforms can effectively help control part of these problems. Yet, the main questions remain as to how this reform should be implemented, and which parts of the health system should be prioritized.

Following certain modifications introduced to the structure of the Ministry of Health and Medical Education after the 11th Iranian presidential elections, the reform of the health sector was seriously taken into account. Along with reforms in treatment and hospital sections, one of the significant issues was to improve the primary care and enhance the PHC system. Because Shiraz (i.e. the capital of Fars province) was a national pilot site for urban family physician since July 14, 2012, Shiraz University of Medical Sciences (SUMS) sought to establish a local model to improve PHC in urban areas. This means believing in PHC in one hand and promoting the role of urban health posts in health development in another. Due to the defects of the previous urban health posts, the new policies concentrated on the renewal of these health posts based on PHC services in urban areas. The policy is called Urban Community Health Center (UCHC), which follows four main goals:

1. Activating urban health posts recognized to be largely inactive;

2. Developing a balance between prevention and treatment as two ultimate purposes, and incorporating them into the functional responsibilities of family physicians;

3. Concentrating on prevention of NCDs in urban areas;

4. Modifying the patterns of providing healthcare services.

According to the scheduled plan, 30 UCHCs in small cities and 8 UCHCs in Shiraz have been built. Each of these centers covers a catchment population of 30,000-50,000. Following this plan, which is managed by family physicians and family health experts, the centers will register families and take active measures to prevent diseases and enhance population health to standard quality. As such, each family member in the area is assigned with one family profile.

Information for each family member is gathered and recorded in specific computerized software. This information includes general items such as number of family members, the status of the family guardian, type of insurance, family physician coverage, and number of ill members. Information further includes specific items about any illness of family members, emphasizing on the diseases which demand primary care intervention; such cases include members with NCDs or psychological disorders, and pregnant women. Once the information is entered into the software, a unique code is assigned to the profile of each of the users. Health experts monitor these data, and after having consultation with a family physician, they will make the decision about services offered to each family member, and if necessary, intervention measures are taken. Any condition is managed according to its specifically defined screening and intervention process. For example, a pregnant woman will be examined by a midwife periodically. Furthermore, patients with cardiac, diabetic, psychological disorders, or other diseases, will be actively followed at primary care level. Consequently, each family member will be taken care of according to their medical needs.

Based on this plan, 30 centers were inaugurated on November 30,2014 . The plan is going through its primary stages. We believe that if the goals of the plan (i.e. UCHC model) are 
well-realized, it can serve as the linchpin in the process of the Iranian health sector reforms. This process, however, needs to be accompanied by sufficient support and critical evaluation. More specifically, experts and policy-makers, assisted health system researchers and intellectuals, should evaluate the whole system.

Finally, because policy-making in the health system faces complex and fuzzy situations, it specifically needs to consider complexity theories and non-linear models. In this direction, policy-makers should consider simple, local, goal-driven and tailored models to control potential challenges, reduce costs, and avoid costly decisions imposed on the health system.

\section{Ethical issues}

Not applicable.

\section{Competing interests}

$\mathrm{MHI}$ has been the founder and director of UCHC model. AKS is a policy analyst for the $\mathrm{UCHC}$ model. MM is a senior assistant and $\mathrm{AH}$ is the executive manager of the UCHC model.

Authors' contributions

All authors contributed equally to writing this letter.

\section{Authors' affiliations}

1Department of Pediatrics, Shiraz University of Medical Sciences, Shiraz, Iran. ${ }^{2}$ Health Policy Research Center, Shiraz University of Medical Sciences, Shiraz, Iran. ${ }^{3}$ Department of Internal Medicine, Shiraz University of Medical Sciences, Shiraz, Iran. ${ }^{4}$ Resource Management Unit of Health System, Shiraz University of Medical Sciences, Shiraz, Iran.

\section{References}

1. Davari M, Haycox A, Walley T. Health care challenges in Iran. Iran J Public Health 2005; 34: 30-1.

2. Lankarani KB, Alavian SM, Peymani P. Health in the Islamic Republic of Iran, challenges and progresses. Med J Islam Repub Iran 2013; 27: 42-9.

3. Merat S, Malekzadeh R. Prevention of non-communicable diseases: what can be done? Arch Iran Med 2013; 16: 136-7.

4. Amini R, Ingman S, Sahaf R. Aging in Iran: Past, Present, and Future. The Journal of Aging in Emerging Economies 2013; 4: 17-34.

5. Sharifi V. Urban mental health in Iran: challenges and future directions. Iran J Psychiatry Behav Sci 2009; 3: 9-14.

6. Joulaei H, Motazedian N. Primary Health Care Strategic Key to Control HIVIAIDS in Iran. Iran J Public Health2013 ; 42: 540-1. 